Artículo científico

Volumen 33(1): Artículo 46141, 2022

e-ISSN 2215-3608, doi:10.15517/am.v33i1.46141

https://revistas.ucr.ac.cr/index.php/agromeso/index

\title{
Efecto sobre el rendimiento del trigo (Triticum aestivum) de la roya amarilla causada por Puccinia striiformis f. sp. tritici $^{1}$
}

\section{Effect on wheat (Triticum aestivum) yield of the yellow rust caused by Puccinia striiformis f. sp. tritici and its effect on yield}

\author{
Miguel Lavilla
}

Recepción: 10 de marzo, 2021. Aceptación: 22 de junio, 2021. Este trabajo formó parte de un proyecto de extensión $\mathrm{N}^{\circ}$ Exp. 2563/17 financiado por la Universidad Nacional del Noroeste de la provincia de Buenos Aires (UNNOBA), Argentina.

2 Universidad Nacional del Noroeste de la provincia de Buenos Aires, Monteagudo 2772 (2700), Pergamino, Buenos Aires, Argentina. miguellavillapergamino@gmail.com (autor para correspondencia, https://orcid.org/0000-0002-7282-4696).

\section{Resumen}

Introducción. En los últimos años la roya amarilla causada por Puccinia striiformis fue la enfermedad más prevalente en trigo (Triticum aestivum), en el norte de la provincia de Buenos Aires. Objetivo. Evaluar el progreso de la roya amarilla en cultivares de trigo, con y sin aplicación de fungicida foliar y su efecto sobre el rendimiento y sus componentes. Materiales y métodos. Este estudio se llevó a cabo durante dos años consecutivos (2017 y 2018) en la localidad de Junín, Buenos Aires, Argentina, en diecinueve cultivares de trigo, con y sin aplicación de fungicida foliar. Se cuantificaron los daños de la roya amarilla sobre el rendimiento y la calidad industrial. Se evaluó la evolución de la enfermedad mediante el área bajo la curva de progreso de la intensidad de la enfermedad (ABCPIE). Se asoció el ABCPIE con el rendimiento, sus componentes y el peso hectolítrico. Resultados. De todas las variables analizadas, el ABCPIE tuvo una interacción significativa $(p<0,05)$ entre la aplicación de fungicida foliar y cultivar utilizado. Los cultivares Lapacho, Minerva, MS 415 y SY 211, fueron resistentes a la roya amarilla. El cultivar más susceptible a la enfermedad fue Algarrobo. La aplicación de fungicida foliar, con niveles bajos de la enfermedad, redujo significativamente su evolución; reflejado a partir del área bajo la curva de progreso de la intensidad de la enfermedad. Conclusión. La evolución en el tiempo de la roya amarilla causada por $P$. striiformis dependió del comportamiento genético del cultivar y de la aplicación de fungicida foliar. Los cultivares que tuvieron los mayores rendimientos, menor ABCPIE y mayor peso hectolítrico fueron: Lapacho, SY 11, BIO 1006 y Minerva.

Palabras claves: enfermedad del follaje, cultivares de trigo, fungicidas foliares.

\begin{abstract}
Introduction. In recent years, yellow rust caused by Puccinia striiformis was the most prevalent disease in wheat (Triticum aestivum), in the north of the province of Buenos Aires, Argentina. Objective. To evaluate the progress of yellow rust in wheat cultivars, with and without foliar fungicide application and its effect on yield and its components. Materials and methods. This study was carried out during two consecutive years (2017 and 2018) in
\end{abstract}


the town of Junín, Buenos Aires, Argentina, on nineteen wheat cultivars, with and without foliar fungicide application. The damages of yellow rust on yield and industrial quality were quantified. Likewise, the evolution of the disease was evaluated using the area under the disease intensity progress curve (ABCPIE). The ABCPIE was associated with the yield, its components, and hectoliter weight. Results. Of all the variables analyzed, the ABCPIE had a significant interaction $(\mathrm{p}<0.05)$ between the foliar fungicide application and the cultivar used. The cultivars Lapacho, Minerva, MS 415, and SY 211 were resistant to yellow rust. The cultivar most susceptible to the disease was Algarrobo. The application of foliar fungicide, with low levels of the disease, significantly reduced its evolution, as reflected from the area under the disease intensity progress curve. Conclusion. The evolution over time of the yellow rust caused by $P$. striiformis depended on the genetic behavior of the cultivar and the application of foliar fungicide. The cultivars that had the highest yields, lowest ABCPIE, and highest hectoliter weight were: Lapacho, SY 11, BIO 1006, and Minerva.

Keywords: foliage disease, wheat cultivars, foliar fungicides.

\section{Introducción}

La producción de trigo (Triticum aestivum) en la Argentina para el año 2020, fue de 21,1 millones de toneladas (Bolsa de cereales, 2021). Las regiones trigueras de la Argentina, subdivididas en: NOA (noroeste argentino), NEA (nordeste argentino), I, II Norte, II Sur, IV, V Norte y V Sur, presentan condiciones climáticas favorables para la aparición de diversas enfermedades. Entre ellas se destacan aquellas que producen síntomas en el follaje como las manchas foliares, los tizones, el oídio, las royas y los mosaicos generados por virus. Estas afectan la fotosíntesis por inducir alteraciones fisiológicas y por reducir el área de tejido verde fotosintéticamente activo. En los últimos años la roya amarilla o estriada, causada por Puccinia striiformis f. sp. tritici, fue la enfermedad más prevalente en el cultivo de trigo en el norte de la provincia de Buenos Aires (región II Sur) (Cordo, 2014).

El hongo P. striiformis es fitopatogénico para la mayoría de los cultivares implantados en la Argentina, debido a la aparición de nuevas razas del patógeno denominadas grupo genético no Warrior (Cluster III) y Warrior (Cluster I) (Hovmøller et al., 2002; Campos, 2017). En términos generales, en la localidad de Junín la raza de roya amarilla predominante es no Warrior (Cluster III) (Campos, 2017). La interacción entre un ambiente predisponente, un hongo fitopatogénico y la siembra de cultivares susceptibles de trigo a las razas previamente citadas de $P$. striiformis, en especial a la no Warrior, provocaron que la roya amarilla sea endémica en el norte de la provincia de Buenos Aires (Barrio et al., 2020).

Las condiciones óptimas para el desarrollo de la enfermedad son las temperaturas entre los $10{ }^{\circ} \mathrm{C}$ y $15^{\circ} \mathrm{C}$ con lluvias intermitentes o rocío (Wiese, 2000).

La pérdida máxima de rendimiento en trigo $(50 \%)$ se produce cuando todo el follaje está infectado con roya amarilla (Shahin et al., 2020; Vergara-Diaz et al., 2015).

El manejo integrado de enfermedades (MIE) es una herramienta de adopción útil para evitar daños por la roya amarilla en el cultivo de trigo. Dentro de los componentes del MIE se encuentran el manejo genético y químico, entre otros.

El objetivo de esta investigación fue evaluar el progreso de la roya amarilla en cultivares de trigo, con y sin aplicación de fungicida foliar, y su efecto sobre el rendimiento y sus componentes. 


\section{Materiales y métodos}

\section{Localización}

Este estudio se llevó a cabo durante dos años consecutivos (2017 y 2018) en la localidad de Junín, Buenos Aires (ruta 188 km 146,5 Lat.: 34²8'58,99”'S; Long.: 6052’36,84”O). Los estudios fitopatológicos, se realizaron en el laboratorio de la Universidad Nacional de Noroeste de la provincia de Buenos Aires, Pergamino, Argentina (UNNOBA).

\section{Diseño experimental}

Se utilizó un diseño experimental en bloques con arreglo factorial 19x2 con tres repeticiones, con aleatorización al azar dentro de los bloques en cada año de estudio. El tamaño de cada unidad experimental fue de 12,6 $\mathrm{m}^{2}$ (6 m de largo x 2,1 m de ancho). Para reducir la variabilidad de los datos, se cosecharon dos submuestras de $2 \mathrm{~m}^{2}$ por repetición, con base en el promedio de ambos como dato a analizar. Los factores a evaluar fueron, factor A) cultivar (C), diecinueve niveles (cultivares de trigo (Triticum aestivum): SY 330, Saeta, Claraz, MS 815, Bio 1006, Ceibo, SN 90, SY 120, SY 211, Serpiente, Huracán, Minerva, MS 116, MS 415, Bio 3006, Basilio, Algarrobo, Baguette 680, y Lapacho); y factor B) fungicida foliar (FF): dos niveles: con y sin FF (bixafen 12,5\%; protioconazole 17,5 $\%$ y trifloxistrobin $15 \%$ ), la dosis aplicada fue de $700 \mathrm{~mL} \mathrm{ha}^{-1}$. La siembra se realizó el 1 de julio de 2017 y de 2018, en forma directa sobre rastrojo de soja a una distancia de $17,5 \mathrm{~cm}$ entre surcos, una densidad de 350 plantas $\mathrm{m}^{-2}$ y fertilizado con $100 \mathrm{~kg} \mathrm{ha}^{-1}$ de fosfato monoamónico. En macollaje se le suministraron $200 \mathrm{~kg} \mathrm{ha}^{-1} \mathrm{de}$ urea. La enfermedad, bajo infecciones naturales, se monitoreó cada semana desde la emergencia del cultivo. La aplicación del FF se realizó en el estado fenológico de encañazón (Z 3.9; Zadoks et al., 1974).

El diagnóstico de la enfermedad y su evolución temporal en los distintos cultivares con y sin la aplicación de FF se determinó con incidencia (INC), severidad (S) e intensidad (INT). Para determinar la INC se eligieron al azar sesenta plantas de cada parcela a las cuales se les diagnosticó la enfermedad. INC (\%): (plantas enfermas / total de plantas muestreadas) * 100 (Agrios, 2005; Julca-Otiniano et al., 2019). La S es el porcentaje de la superficie del órgano enfermo, ya sea de hojas, tallos, raíces o frutos afectados por la enfermedad y varía entre 0 y 100 . S (\%): [área de tejido enfermo * área total (sano + enfermo)-1] * 100 (Agrios, 2005). El INT involucra tanto a la INC como a la $\mathrm{S}$ de la enfermedad. La INT de la roya amarilla amplía el criterio para la toma de decisiones en cuanto a la aplicación de una medida fitosanitaria y se calcula como: INT (\%): (INC * S) / 100.

\section{Cálculo del área bajo la curva del progreso de la intensidad de la enfermedad}

La determinación del área bajo la curva de progreso de la enfermedad (ABCPE) es una técnica de análisis recomendada cuando se requiere identificar las relaciones del progreso de la enfermedad y el período de duración del área foliar, respecto al impacto sobre el rendimiento. Este método de análisis epifitiológico no necesita tipo de ajuste a modelos predeterminados $\mathrm{y}$, por ende, no requiere de consecuentes transformaciones de valores que puedan enmascarar o confundir posibles efectos de tratamiento. Sin embargo, valores bajos de la enfermedad, durante el período de monitoreo, tienen poco efecto sobre el ABCPE.

El ABCPE estabiliza la varianza de los valores porcentuales de la enfermedad dentro de los tratamientos. Es posible detectar efectos de los mismos que podrían no detectarse por el análisis de tasas relativas de incremento de la enfermedad. 
Los resultados pueden ser más confiables cuando, al reducir la varianza, disminuye el coeficiente de variación, el cual por lo regular es alto en este tipo de estudios e incrementa la confiabilidad de las conclusiones derivadas de este tipo de estudios epidemiológicos.

El ABCPE se calcula al integrar los rectángulos formados por el punto medio de la incidencia y de la severidad de la enfermedad alcanzada entre diferentes tiempos en que se monitorea.

Fórmula de ABCPE:

$\sum_{\mathrm{i}}\left(\left(\mathrm{Y}_{\mathrm{i}}+\mathrm{Y}_{\mathrm{i}+1}\right) *\left(\mathrm{t}_{\mathrm{i}+1}-\mathrm{ti}\right)\right) / 2$

Donde Yi es la incidencia o severidad de la enfermedad y t es el período de evaluación en días después de la siembra o cualquier otra escala que se desee usar en función del tiempo. En este caso las unidades serán porcentajes (\%) y días. Además, este método de análisis epidémico considera la variación de la epidemia en el tiempo, para los análisis comparativos visuales que pudieran requerirse.

Para calcular el área bajo la curva del progreso de la intensidad de la enfermedad (ABCPIE), se evaluó la severidad e incidencia de la roya amarilla en el cultivo de trigo durante cinco semanas posteriores al tratamiento de FF. El ABCPIE se calculó de la siguiente forma: [Área bajo la curva del progreso de la severidad de la enfermedad (ABCPEs) * Área bajo la curva del progreso de la incidencia de la enfermedad (ABCPEi)]/100, el cual fue utilizado como variable para analizar el progreso de la enfermedad en cada tratamiento. El ABCPEs se calculó con los datos de severidad de la roya amarilla recopilados en las cinco semanas de evaluación y el ABCPEi con los datos de incidencia obtenidos en el mismo período de tiempo.

\section{Rendimiento y sus componentes}

Se cosechó en forma manual una muestra de $2 \mathrm{~m}^{2}$ por tratamiento y repetición. A partir de la muestra se determinaron los componentes del rendimiento de la siguiente manera:

- Espigas $\mathrm{m}^{-2}\left(\mathrm{E} \mathrm{m}^{-2}\right)$ : número de espigas en los $2 \mathrm{~m}^{2}$ de cada repetición por tratamiento.

- Espiguillas/espiga (Esp./E): se recolectaron veinte espigas al azar de cada repetición por tratamiento y se cuantificó el número de espiguillas por espiga.

- Granos/espiga (G/E): de las 20 espigas recolectadas se cuantificó el número de granos por espiga.

- Granos $\mathrm{m}^{-2}\left(\mathrm{NG} \mathrm{m}^{-2}\right)$ : se determinaron al relacionar el peso total de la muestra con el peso de mil granos (P1000) en gramos.

- P1000: se pesaron en una balanza de precisión 1000 granos de cada tratamiento y repetición.

- Rendimiento (R) en $\mathrm{kg} \mathrm{ha}^{-1}$ : los granos post trilla y zarandeo se pesaron en una balanza de precisión, para calcular el rendimiento para cada tratamiento y repetición.

\section{Parámetro de calidad industrial}

- Peso hectolítrico (PH) en $\mathrm{kg} \mathrm{hL}^{-1}$ : se calculó con un equipamiento denominado Higrómetro HD-1021USB - Delver S.A. para cada tratamiento y repetición. El procedimiento consta de ubicar los granos de trigo en la cavidad de higrómetro hasta cubrirlo completamente, luego el higrómetro mediante un algoritmo determina el peso hectolítrico de la muestra de trigo. 


\section{Análisis estadísticos}

Los datos obtenidos para rendimiento y sus componentes, al igual que la calidad industrial y el ABCPIE, se estudiaron mediante un análisis de la varianza y la comprobación de supuestos teóricos correspondientes. Las comparaciones de medias se realizaron a través de la prueba de DGC (Di-Rienzo, Guzmán y Casanoves), con un nivel de significancia de 0,05 (Di-Rienzo et al., 2011).

Se correlacionó el rendimiento, sus componentes, el ABCPIE y el peso hectolítrico a través de una correlación de Pearson (Rice, 1995).

Los análisis estadísticos se realizaron con el programa InfoStat (Di-Rienzo et al., 2011).

\section{Resultados}

La aplicación de fungicida foliar (FF) incrementó a nivel significativo $(\mathrm{p}<0,05)$ las espigas por $\mathrm{m}^{2}$, las espiguillas por espiga, el peso de mil granos y el peso hectolítrico (Cuadro 1). Respecto al control de la roya amarilla, la aplicación de FF tuvo un efecto significativo $(\mathrm{p}<0,05)$ en la reducción del área bajo la curva de progreso de la intensidad de la enfermedad (ABCPIE) y disminuyó su evolución en un 76 \% (Cuadro 1).

Cuadro 1. Rendimiento (R), sus componentes, el área bajo la curva de progreso de la intensidad de la enfermedad (ABCPIE) de la roya amarilla (Puccinia striiformis) bajo infecciones naturales y el peso hectolítrico (PH), asociados a la aplicación de fungicida foliar (FF), en cultivares de trigo (Triticum aestivum). Junín, Buenos Aires, Argentina. 2017-2018.

Table 1. Yield (R), its components, the area under the disease intensity progress curve (ABCPIE) of yellow rust (Puccinia striiformis) under natural infections and the hectoliter weight $(\mathrm{PH})$ associated with the foliar fungicide (FF) application in wheat cultivars. Junín, Buenos Aires, Argentina. 2017-2018.

\begin{tabular}{|c|c|c|c|c|c|c|c|c|c|c|c|c|c|c|c|c|}
\hline $\begin{array}{l}\text { Aplicación } \\
\text { de FF }\end{array}$ & \multicolumn{2}{|c|}{$\mathbf{E} \mathbf{~ m}^{-2}$} & \multicolumn{2}{|c|}{ Esp./E } & \multicolumn{2}{|c|}{ G/Esp. } & \multicolumn{2}{|c|}{ NG $\mathbf{m}^{-2}$} & \multicolumn{2}{|c|}{ P1000 } & \multicolumn{2}{|c|}{$\mathbf{R}\left(\mathrm{kg} \mathrm{ha}^{-1}\right)$} & \multicolumn{2}{|c|}{ ABCPIE } & \multicolumn{2}{|c|}{ PH } \\
\hline $\mathrm{C} / \mathrm{FF}$ & 481 & A & 14 & A & 3 & A & 11444 & A & 34 & A & 3903 & A & 64 & A & 73 & A \\
\hline $\mathrm{S} / \mathrm{FF}$ & 437 & B & 13 & B & 3 & A & 10495 & A & 32 & B & 3411 & B & 272 & B & 72 & B \\
\hline
\end{tabular}

$\mathrm{E} \mathrm{m}^{-2}$ : espigas por $\mathrm{m}^{2}$, Esp./E: espiguillas por espiga, G/Esp.: granos por espiguilla, $\mathrm{NG}^{-2}$ : número de granos por $\mathrm{m}^{2}$, P1000: peso de mil granos en gramos, C/FF: con fungicida foliar, S/FF: sin fungicida foliar, FF: fungicida foliar, ABCPIE: área bajo la curva del progreso de la intensidad de la enfermedad, $\mathrm{PH}$ : peso hectolítrico. / $\mathrm{E} \mathrm{m}^{-2}$ : spikes per $\mathrm{m}^{2}$, Esp./E: spikelets per spike, G/Esp.: grains per spikelet, $\mathrm{NG} \mathrm{m}^{-2}$ : number of grains per $\mathrm{m}^{2}, \mathrm{P} 1000$ : weight of one thousand grains in grams, $\mathrm{C} / \mathrm{FF}$ : with foliar fungicide, S/FF: without foliar fungicide. FF: foliar fungicide, ABCPIE: area under the disease intensity progress curve, PH: test weight.

Las variedades presentaron diferencias significativas para todos los subcomponentes y componentes de rendimiento (Cuadro 2). Los genotipos que tuvieron los mayores rendimientos fueron: Lapacho, SY 120, SY 211, SAETA, BIO 1006, MS 415, SY 330, BIO 3006, Minerva y MS 116 (Cuadro 2). Los materiales con menor ABCPIE fueron: Lapacho, SY 211, MS 415, Minerva, BIO 1006 y MS 815. Los cultivares que tuvieron mayor peso hectolítrico fueron: Minerva, SAETA, MS 415, BIO 1006, Claraz, MS 116, SY 211, Huracán, BIO 3006 y Lapacho. Los cultivares que tuvieron los mayores rendimientos, menor ABCPIE y mayor peso hectolítrico fueron: Lapacho, SY 11, BIO 1006 y Minerva. Estos últimos cultivares deberían ser considerados como los más adaptados a ambientes con roya amarilla, debido a que presentaron resistencia a la enfermedad, altos rendimiento y los granos presentaron un elevado peso hectolítrico. 
Cuadro 2. Rendimiento (R), sus componentes, el área bajo la curva de progreso de la intensidad de la enfermedad (ABCPIE) de la roya amarilla (Puccinia striiformis) bajo infecciones naturales y el peso hectolítrico ( $\mathrm{PH}$ ) asociados con los diferentes cultivares de trigo (Triticum aestivum) evaluados. Junín, Buenos Aires, Argentina. 2017-2018.

Table 2. Yield (R), its components, the area under the disease intensity progress curve (ABCPIE) of yellow rust (Puccinia striiformis) under natural infections and the hectoliter weight $(\mathrm{PH})$ associated with different cultivars of wheat (Triticum aestivum) evaluated. Junín, Buenos Aires, Argentina. 2017-2018.

\begin{tabular}{|c|c|c|c|c|c|c|c|c|c|c|c|c|c|c|c|c|}
\hline \multirow{2}{*}{$\begin{array}{l}\text { Cultivar } \\
\text { Algarrobo }\end{array}$} & \multicolumn{2}{|c|}{$E \mathbf{m}^{-2}$} & \multicolumn{2}{|c|}{ Esp./E } & \multicolumn{2}{|c|}{ G/Esp. } & \multicolumn{2}{|c|}{$\mathbf{N G} \mathbf{m}^{-2}$} & \multicolumn{2}{|c|}{ P1000 } & \multicolumn{2}{|c|}{$\mathbf{R}\left(\mathrm{kg} \mathrm{ha}^{-1}\right)$} & \multicolumn{2}{|c|}{ ABCPIE } & \multicolumn{2}{|c|}{ PH } \\
\hline & 447 & B & 14 & A & 3 & A & 11523 & A & 30 & A & 3528 & A & 735 & G & 68 & A \\
\hline Baguette 680 & 429 & B & 13 & A & 3 & A & 11507 & A & 29 & A & 3429 & A & 166 & $\mathrm{C}$ & 69 & A \\
\hline Basilio & 317 & A & 13 & A & 3 & B & 9815 & $\mathrm{~A}$ & 29 & A & 2943 & A & 214 & $\mathrm{D}$ & 65 & A \\
\hline BIO 1006 & 400 & B & 13 & A & 3 & A & 10369 & A & 40 & $\mathrm{C}$ & 4163 & B & 8 & A & 75 & B \\
\hline BIO 3006 & 418 & B & 15 & A & 3 & B & 9515 & A & 39 & $\mathrm{C}$ & 3774 & B & 132 & $\mathrm{C}$ & 74 & B \\
\hline Ceibo & 500 & $\mathrm{C}$ & 13 & A & 2 & A & 10354 & $\mathrm{~A}$ & 31 & A & 3326 & A & 161 & $\mathrm{C}$ & 71 & A \\
\hline Claraz & 593 & $\mathrm{C}$ & 13 & A & 3 & A & 9699 & A & 33 & A & 3238 & A & 219 & $\mathrm{D}$ & 75 & B \\
\hline Huracán & 417 & B & 13 & A & 3 & A & 9889 & A & 32 & A & 3193 & A & 263 & $\mathrm{E}$ & 74 & B \\
\hline Lapacho & 468 & B & 13 & A & 3 & A & 14830 & $\mathrm{~B}$ & 31 & A & 4649 & B & 0 & A & 73 & B \\
\hline Minerva & 396 & B & 14 & A & 3 & A & 11428 & A & 33 & A & 3749 & B & 0 & A & 81 & B \\
\hline MS 116 & 374 & B & 13 & A & 2 & A & 10786 & A & 34 & B & 3673 & B & 291 & $\mathrm{E}$ & 75 & B \\
\hline MS 415 & 450 & B & 13 & A & 3 & $\mathrm{~A}$ & 10882 & A & 37 & $\mathrm{~B}$ & 4001 & B & 0 & A & 77 & B \\
\hline MS 815 & 579 & $\mathrm{C}$ & 13 & A & 2 & A & 8495 & A & 32 & A & 2763 & A & 19 & A & 72 & A \\
\hline SAETA & 533 & $\mathrm{C}$ & 13 & A & 3 & A & 11912 & A & 36 & B & 4256 & B & 101 & $\mathrm{~B}$ & 78 & B \\
\hline Serpiente & 417 & B & 14 & A & 3 & A & 7511 & A & 31 & A & 2321 & A & 144 & $\mathrm{C}$ & 69 & A \\
\hline SN 90 & 524 & $\mathrm{C}$ & 13 & A & 2 & A & 11938 & A & 28 & $\mathrm{~A}$ & 3396 & A & 351 & $\mathrm{~F}$ & 68 & A \\
\hline SY 120 & 569 & $\mathrm{C}$ & 15 & A & 3 & $\mathrm{~B}$ & 14492 & B & 32 & A & 4601 & B & 83 & $\mathrm{~B}$ & 70 & A \\
\hline SY 211 & 511 & $\mathrm{C}$ & 13 & B & 3 & A & 12490 & A & 36 & B & 4560 & B & 0 & A & 74 & B \\
\hline SY 330 & 383 & B & 13 & B & 3 & A & 10982 & A & 36 & B & 3921 & B & 310 & $\mathrm{E}$ & 71 & A \\
\hline
\end{tabular}

$\mathrm{E} \mathrm{m}^{-2}$ : espigas por $\mathrm{m}^{2}$, Esp./E: espiguillas por espiga, G/Esp.: granos por espiguilla, $\mathrm{NG} \mathrm{m}^{-2}$ : número de granos por $\mathrm{m}^{2}$, P1000: peso de mil granos en gramos, ABCPIE: área bajo la curva del progreso de la intensidad de la enfermedad, PH: Peso hectolítrico. / E m²: spikes per $\mathrm{m}^{2}$, Esp./E: spikelets per spike, $\mathrm{G} /$ Esp.: grains per spikelet, $\mathrm{NG} \mathrm{m}^{-2}$ : number of grains per $\mathrm{m}^{2}, \mathrm{P} 1000$ : weight of one thousand grains in grams, ABCPIE: area under the curve of the progress of the intensity disease, PH: test weight.

De todas las variables analizadas, el ABCPIE tuvo una interacción significativa $(\mathrm{p}<0,05)$ entre la aplicación de fungicida foliar y el cultivar utilizado. Los cultivares Lapacho, Minerva, MS 415 y SY 211, presentaron resistencia frente a la roya amarilla y la aplicación de FF en estos cultivares, para el ambiente y los años en donde se llevó a cabo el estudio, no presentó reducciones significativas $(\mathrm{p}<0,05)$ en el ABCPIE (Cuadro 3).

El rendimiento en $\mathrm{kg} \mathrm{ha}^{-1}$, presentó una correlación positiva y significativa $(\mathrm{p}<0,05)$ con todos los componentes y con el $\mathrm{pH}$ (Cuadro 4). Las mayores correlaciones del rendimiento fueron con el número de granos por $\mathrm{m}^{2}$ $(0,92)$ y con el peso de mil granos $(0,56)$. El peso hectolítrico también se correlacionó de manera positiva $(0,42)$ y significativa $(\mathrm{p}<0,05)$ con el rendimiento del cultivo de trigo. Asimismo, hubo una correlación negativa y significativa $(\mathrm{p}<0,05)$ entre el rendimiento y el ABCPIE $(-0,3)$. La roya amarilla redujo el rendimiento al afectar los componentes de rendimiento; además, afectó de manera negativa el peso hectolítrico. 
Cuadro 3. Área bajo la curva de progreso de la intensidad de la enfermedad (ABCPIE) causada por la roya amarilla (Puccinia striiformis) bajo infecciones naturales, asociada con los diferentes cultivares de trigo (Triticum aestivum) evaluados. Junín, Buenos Aires, Argentina. 2017-2018.

Table 3. Area under the disease intensity progress curve (ABCPIE) caused by yellow rust (Puccinia striiformis) under natural infections, associated with different cultivars of wheat (Triticum aestivum) evaluated. Junín, Buenos Aires, Argentina. 2017-2018.

\begin{tabular}{|c|c|c|}
\hline \multirow[b]{2}{*}{ Cultivar } & \multicolumn{2}{|c|}{ ABCPIE } \\
\hline & $\mathrm{C} / \mathrm{FF}$ & S/FF \\
\hline Algarrobo & $386,73 \quad E$ & $1083,79 \mathrm{H}$ \\
\hline Baguette 680 & $44,02 \mathrm{~A}$ & $287,96 \mathrm{D}$ \\
\hline Basilio & 88,47 B & $338,94 E$ \\
\hline BIO 1006 & $3,75 \mathrm{~A}$ & $12,43 \mathrm{~A}$ \\
\hline BIO 3006 & 46,98 A & $216,31 \mathrm{C}$ \\
\hline Ceibo & 56,66 A & $264,98 \mathrm{D}$ \\
\hline Claraz & $66,31 \mathrm{~A}$ & $371,85 \quad E$ \\
\hline Huracán & $50,08 \mathrm{~A}$ & $476,03 \mathrm{~F}$ \\
\hline Lapacho & $0 \mathrm{~A}$ & $0 \mathrm{~A}$ \\
\hline Minerva & $0 \mathrm{~A}$ & $0 \mathrm{~A}$ \\
\hline MS 116 & $97,07 \quad \mathrm{~B}$ & $485,3 \quad \mathrm{~F}$ \\
\hline MS 415 & $0 \mathrm{~A}$ & $0 \mathrm{~A}$ \\
\hline MS 815 & $6,91 \mathrm{~A}$ & 31,76 A \\
\hline Saeta & $32,36 \mathrm{~A}$ & $169,15 \mathrm{C}$ \\
\hline Serpiente & $93,51 \quad \mathrm{~B}$ & $193,86 \mathrm{C}$ \\
\hline SN 90 & $199,41 \mathrm{C}$ & $501,76 \mathrm{~F}$ \\
\hline SY 120 & $32,26 \quad \mathrm{~A}$ & $133,09 \quad \mathrm{~B}$ \\
\hline SY 211 & $0 \mathrm{~A}$ & $0 \mathrm{~A}$ \\
\hline SY 330 & $20,12 \mathrm{~A}$ & $599,09 \quad \mathrm{G}$ \\
\hline
\end{tabular}

C/FF: con fungicida foliar, S/FF: sin fungicida foliar. / C/FF: with foliar fungicide, S/FF: without foliar fungicide.

Cuadro 4. Matriz de correlación de Pearson entre las variables analizadas. Roya amarilla causada por Puccinia striiformis, bajo inoculaciones naturales en cultivares de trigo (Triticum aestivum). Junín, Buenos Aires, Argentina. 2017-2018.

Table 4. Pearson's correlation matrix between the variables analyzed. Yellow rust caused by Puccinia striiformis, under natural inoculations in wheat cultivars (Triticum aestivum). Junín, Buenos Aires, Argentina. 2017-2018.

\begin{tabular}{|c|c|c|c|c|c|c|c|c|}
\hline & $\mathbf{R}\left(\mathrm{kg} \mathrm{ha}^{-1}\right)$ & $\mathbf{E ~ m}^{-2}$ & Esp./E & G/Esp. & P1000 & NG m $^{-2}$ & PH $\left(k g h L^{-1}\right)$ & ABCPIE \\
\hline $\mathbf{R}\left(\mathrm{kg} \mathrm{ha}^{-1}\right)$ & 1 & 0,01 & 0,00032 & 0,00190 & 0,00000014 & 0,0001 & 0,00017 & 0,01 \\
\hline $\mathbf{E ~ m}^{-2}$ & 0,31 & 1 & 0,003 & 0,86 & 0,28 & 0,01 & 0,19 & 0,1 \\
\hline Esp./E & 0,4 & 0,34 & 1 & 0,0000120 & 0,0013 & 0,01 & 0,19 & 0,41 \\
\hline G/Esp. & 0,35 & $-0,02$ & 0,48 & 1 & 0,04 & 0,01 & 0,15 & 0,91 \\
\hline P1000 & 0,56 & 0,12 & 0,36 & 0,24 & 1 & 0,09 & 0,000000011 & 0,00017 \\
\hline NG $\mathbf{m}^{-2}$ & 0,92 & 0,31 & 0,32 & 0,29 & 0,2 & 1 & 0,06 & 0,16 \\
\hline PH $\left(\mathbf{k g ~ h L ^ { - 1 } )}\right.$ & 0,42 & 0,15 & 0,15 & 0,17 & 0,6 & 0,22 & 1 & 0,00021 \\
\hline ABCPIE & $-0,3$ & $-0,19$ & $-0,1$ & $-0,01$ & $-0,42$ & $-0,16$ & $-0,41$ & 1 \\
\hline
\end{tabular}

$\mathrm{R}\left(\mathrm{kg} \mathrm{ha}^{-1}\right)$ : rendimiento, $\mathrm{E} \mathrm{m}^{-2}$ : espigas por $\mathrm{m}^{2}$, Esp./E: espiguillas por espiga,G/Esp.: granos por espiguilla, P1000: peso de mil granos en gramos, $\mathrm{NG} \mathrm{m}^{-2}$ : número de granos por $\mathrm{m}^{2}, \mathrm{PH}\left(\mathrm{kg} \mathrm{hl}^{-1}\right)$ : peso hectolítrico, ABCPIE: área bajo la curva de progreso de la intensidad de la enfermedad. / $\mathrm{R}\left(\mathrm{kg} \mathrm{ha}^{-1}\right)$ : yield, $\mathrm{E} \mathrm{m}^{-2}$ : spikelets per $\mathrm{m}^{2}$, Sp./E: spikelets per spike, G / Sp.: grains per spikelet, P1000: weight of one thousand grains in grams, $\mathrm{NG} \mathrm{m}^{-2}$ : number of grains per $\mathrm{m}^{2}, \mathrm{PH}\left(\mathrm{kg} \mathrm{hl}^{-1}\right)$ : hectoliter weight, ABCPIE: area under the curve of the progress of the intensity disease. 


\section{Discusión}

La utilización de fungicidas foliares en cualquiera de los cultivares aumentó el rendimiento de manera significativa en un $14 \%$ (Cuadro 1). Las pérdidas de rendimiento alcanzan hasta un $50 \%$ en cultivares susceptibles, cuando los mismos no son tratados con fungicidas foliares (Vergara-Diaz et al., 2015).

En este estudio, los genotipos de trigo presentaron diferentes comportamientos frente a la roya amarilla. El cultivar Algarrobo fue el de mayor susceptibilidad a infecciones naturales de roya amarilla (ABCPIE: 735; Cuadro 2).

El rendimiento en granos y el peso de 1000 granos se redujo significativamente en aquellos ambientes sin fungicida foliar y con la implantación de cultivos susceptibles a la roya amarilla; por ejemplo, en el cultivar Algarrobo sin aplicación de fungicida foliar, el rendimiento se redujo en casi un $75 \%$ (Cuadro 1). Otros investigadores en Egipto observaron pérdidas de rendimiento de hasta un 69,33\%, por causa de la roya amarilla en cultivares susceptibles (Shahin et al., 2020).

El incremento del ABCPIE redujo significativamente el rendimiento en granos y el peso de 1000 granos con un coeficiente de correlación de Pearson de $-0,3$ y $-0,42$ respectivamente (Cuadro 4). Estos resultados coinciden con los de Shahin et al. (2020), quienes observaron que incrementos en el área bajo la curva de progreso de la enfermedad redujo significativamente el rendimiento y el peso de 1000 granos con un coeficiente de correlación $\left(\mathrm{R}^{2}\right)$ de 0,974 y 0,928 , respectivamente.

La roya amarilla no solo causa pérdidas del rendimiento en granos, en esta experiencia pudo corroborarse que el peso hectolítrico se redujo significativamente (coeficiente de correlación de Pearson -0,41; Cuadro 4), cuando aumentó el ABCPIE de la roya amarilla del trigo. Estos resultados coinciden con los de O'Brien et al., (1990) en Australia, en donde revelaron reducciones significativas del peso hectolítrico de los granos en cultivares susceptibles a la roya amarilla.

\section{Conclusiones}

La evolución en el tiempo de la roya amarilla causada por $P$. striiformis dependió del comportamiento genético del cultivar y de la aplicación de fungicida foliar. Los cultivares que tuvieron los mayores rendimientos, menor ABCPIE y mayor peso hectolítrico fueron: Lapacho, SY 11, BIO 1006 y Minerva.

\section{Referencias}

Agrios, G. N. (2005). Plant pathology (5 ${ }^{\text {th }}$ Ed.). Academic Press.

Barrio, M., Lavilla, M. A., Beltrán, G., Ivancovich, A., \& Pepper, A. (2020). Evaluación del momento óptimo de aplicación de fungicida foliar para el control de roya amarilla. Revista Técnica Cultivos de Invierno, 2020, 107-111.

Bolsa de cereales. (2021, 18 de febrero). Estimaciones agrícolas. https://www.bolsadecereales.com/estimaciones-informes

Campos, P. E. (2017). Identificación de razas exóticas de roya amarilla en región triguera Argentina. Instituto Nacional de Tecnología Agropecuaria.

Di-Rienzo, J. A., Casanoves, F., Balzarini, M. G., González, L., Tablada, M., \& Robledo, C. W. (2011). InfoStat. Grupo InfoStat. http://www.infostat.com.ar

Cordo, C. A. (2014). Enfermedades del trigo. Avances científicos en la Argentina. Editorial de la Universidad de La Plata. 
Hovmøller, M. S., Justesen, A. F., \& Brown, J. K. M. (2002). Clonality and long-distance migration of Puccinia striiformis f.sp. tritici in north-west Europe. Plant Pathology, 51(1), 24-32. https://doi.org/10.1046/j.1365-3059.2002.00652.x

Julca-Otiniano, A., Borjas Ventura, R., Alvarado Huamán, L., Julca Vera, N., Castro Cepero, V., \& Bello Ames, S. (2019). Relación entre la incidencia y la severidad de la roya del café (Hemileia vastatrix) en San Ramón, Chanchamayo, Perú. Journal of Science and Research, 4(4), 1-9. https://doi.org/10.5281/zenodo.3477556

O’Brien, L., Brown, J. S., Panozzo, J. F., \& Archer, M. J. (1990). The effect of stripe rust on the quality of Australian wheat varieties. Australian Journal of Agricultural Research, 41(5), 827-833. https://doi.org/10.1071/ar9900827

Rice, J. A. (1995). Mathematical statistics and data analysis. ( $2^{\text {nd }}$ Ed.). Duxbury Press.

Shahin, A., Ashmawy, M., El-Orabey, W., \& Esmail, S. (2020). Yield losses in wheat caused by stripe rust (Puccinia striiformis) in Egypt. American Journal of Life Sciences, 8(5), 127-134. https://doi.org/10.11648/j.ajls.20200805.17

Vergara-Diaz, O., Kefauver, S. C., Elazab, A., Nieto-Taladriz, M. T., \& Araus, J. L. (2015). Grain yield losses in yellow-rusted durum wheat estimated using digital and conventional parameters under field conditions. The Crop Journal, 3(3), 200-210. https://doi.org/10.1016/j.cj.2015.03.003

Wiese, M. V. (2000). Compendio de enfermedades del trigo. Hemisferio Sur Editorial.

Zadoks, J. C., Chang, T. T., \& Konzak, C. F. (1974). A decimal code for the growth stages of cereals. Weed Research, 14(6), 415-421. https://doi.org/10.1111/j.1365-3180.1974.tb01084.x 